\title{
Polymeric nanoparticles with a sera-derived coating for efficient cancer cell uptake and killing
}

\author{
Daniel Nierenberg ${ }^{1,5}$, Orielyz Flores ${ }^{1,5}$, David Fox ${ }^{2,4}$, Yuen Yee Li Sip ${ }^{2,3}$, Caroline Finn ${ }^{1}$, \\ Heba Ghozlan ${ }^{1}$, Amanda Cox ${ }^{1}$, K. Kai McKinstry ${ }^{1}$, Lei Zhai ${ }^{2,3,4}$ and Annette R Khaled ${ }^{1 *}$
}

\begin{abstract}
${ }^{1}$ Burnett School of Biomedical Sciences, College of Medicine, University of Central Florida, Orlando, FL, USA; ${ }^{2}$ Nanotechnology Science Center, University of Central Florida, Orlando, FL, USA; ${ }^{3}$ Department of Materials Science and Engineering, College of Engineering and Computer Science, University of Central Florida, Orlando, FL, USA; ${ }^{4}$ Department of Chemistry, College of Science, University of Central Florida, Orlando, FL, USA; ${ }^{5}$ These authors contributed equally.

*Corresponding author
\end{abstract}




\section{Supplemental Methods}

\section{DiI dye and Taxol encapsulation characterization}

To verify uniform DiI dye and taxol encapsulation in COOH-HBPE-NPs and PEGHBPE-NPs, fluorescence and absorbance quantification for DiI and taxol, respectively, was performed. For evaluating DiI dye encapsulation, a calibration curve was established in $50 \mu \mathrm{l}$ of PBS initially with $1 \mu \mathrm{g}$ of unencapsulated DiI, and serially diluted by half, up to $00.7813 \mu \mathrm{g}$ of DiI. Additionally, $10 \mu \mathrm{g}$ of non-PEGylated or PEGylated DiIencapsulated NPs were dispensed in an $\mathrm{HCl}$-acidified $50 \mu \mathrm{PBS}$ solution $(\mathrm{pH}=4)$ and incubated for 6 hours at room temperature to ensure the release of encapsulated DiI through HBPE polymer ester hydrolysis. The acidified NP solutions and serial dilutions of unencapsulated DiI were then dispensed in a 96-well plate. DiI fluorescence was then quantified with a Cytation 5 Cell Imaging Multi-Mode Reader. Fluorescence measurements were performed in triplicate at $531 \mathrm{~nm}$ excitation and $593 \mathrm{~nm}$ emission wavelengths. A trendline equation of the serial dilutions was then created through Microsoft Excel software. The estimated encapsulated DiI concentration in COOHHBPE-NPs and PEG-HBPE-NPs was calculated by substituting the trendline's y-axis value for a NP's fluorescence value, and solving for the trendline's slope value, X. For assessing taxol encapsulation, a calibration curve was established in $1 \mathrm{ml}$ of PBS initially with $87.5 \mu \mathrm{g}$ of unencapsulated taxol, and serially diluted by half, up to $5.4687 \mu \mathrm{g}$ of taxol. Furthermore, $100 \mu \mathrm{g}$ of non-PEGylated or PEGylated taxol-encapsulated NPs were incubated in an $\mathrm{HCl}$-acidified $1 \mathrm{ml} \mathrm{PBS}$ solution $(\mathrm{pH}=4)$ for 6 hours at room temperature to verify taxol release of encapsulated taxol via polymer hydrolysis. The 
acidified NP solutions and serial dilutions of unencapsulated taxol were then placed in a UV Cuvette (Fisher Scientific) and taxol absorbance was read at a $250 \mathrm{~nm}$ wavelength using a Beckman Coulter DU 800 Spectrophotometer (Brea, CA, USA). A trendline equation of the serial dilutions and estimation of encapsulated taxol concentration in COOH-HBPE-NPs and PEG-HBPE-NPs was performed in the same fashion as the DiI measurements, with the exception of the trendline's y-axis value being substituted for absorbance, rather than fluorescence.

\section{Assessment of Protein Corona by Gel Electrophoresis}

To isolate and assess coronal proteins associated with HBPE-NPs a $5: 1$ or $20: 1$

volumetric ratio of NP to mouse serum was used. HBPE-NPs were incubated with normal mouse sera (NS) for 1 hour under gentle agitation at room temperature. Nanoparticle:sera samples were centrifuged three times at 17,000 $\mathrm{x}$ g for 30 minutes using PBS as a washing solution and an Optima TLX ultracentrifuge (Beckman Coulter, Brea, CA, USA) for pelleting nanomaterials. After the third round of centrifugation of NS-coated HBPENP samples, pellets were resuspended in PBS. The protein concentration of resuspended samples was then measured in triplicate using a Nanodrop 8000 (ThermoFisher Scientific). Approximately $0.2 \mathrm{mg} / \mathrm{ml}$ of nanoparticle:sera samples were treated with a final concentration of $20 \mathrm{mM} \beta$-mercaptoethanol and boiled for 5 minutes at $90^{\circ} \mathrm{C}$ to detach coronal proteins from NPs. Boiled samples were then spun down briefly at 16,000 $\mathrm{x}$ g using a 5415R centrifuge (Eppendorf) and dispensed in a $15 \mu 1$-per-well Mini-Protean TGX polyacrylamide gel. Gels were run at $80 \mathrm{~V}$ for 2.5 hours. A reference Precision Plus 
Protein Dual Color Standards protein ladder was used with each gel. After

electrophoresis, gels were stained with Coomassie Brilliant Blue G-250 and imaged with an Odyssey infrared imaging system (Li-Cor Biosciences, Lincoln, NE, USA) or ChemiDoc MP imaging system (Bio-Rad Laboratories), for 5:1 and 20:1 volumetric ratio, respectively.

\section{Supplemental Figure Legends.}

\section{Supplemental Figure 1. COOH-HBPE-NPs and PEG-HBPE-NPs are equivalently}

loaded with cargo. NPs were treated with $\mathrm{HCl}$-acidified $50 \mu \mathrm{PBS}$ solution (pH 4) to release cargo upon polymer degradation after hydrolysis. (A) Calibration curve was generated for serial dilutions of DiI as described in methods and read in a Cytation 5 multimodal plate reader at at $531 \mathrm{~nm}$ excitation and $593 \mathrm{~nm}$ emission wavelengths. Table summaries average fluorescence of DiI from $10 \mathrm{ug}$ of hydrolyzed NPs in $50 \mathrm{ul}$ volume and estimates DiI concentration in a typical $10 \mathrm{ul}$ dose of HBPE-NPs. (B) Representative example of absorbance spectrum for NPs loaded with taxol and treated as above to release cargo. Absorbance was read at a $250 \mathrm{~nm}$ wavelength (UV/Vis) using a Beckman Coulter DU 800 Spectrophotometer. Tables show average absorbance for taxol released from $100 \mathrm{ug}$ of NPs in $1 \mathrm{ml}$ volume and the concentration of taxol estimated to be loaded in $0.01 \mathrm{mg}$ polymer (in $1 \mathrm{ul}$ ) dose of HBPE-NPs based on the calibration curve generated from taxol serial dilutions. 
Supplemental Figure 2. Protein profiles are associated with coronae formed on HBPE-NPs treated with normal mouse sera (NS). Proteins absorbed by COOHHBPE-NPs treated with NS was assessed by SDS-PAGE (sodium dodecyl sulfate polyacrylamide gel electrophoresis) gels and visualized by Coomassie staining. $\mathrm{COOH}-$ HBPE-NPs were treated with sera at a volume:volume ratio of 5:1 or 20:1. A PrecisionPlus Protein Dual Color protein reference ladder was used for molecular weight (MW) comparison.

Supplemental Figure 3. HBPE-NPs pre-treated with NS show inreassed uptake by cancer cells. Representative Cytation 5 microscopic images taken $24 \mathrm{~h}$ post-treatment of MDA-MB-231 (left column), HUVEC (middle column), and THP-1 (right column) cells treated with DiI dye-encapsulated COOH-HBPE-NPs (top row), PEG-HBPE-NPs (middle row), and COOH-HBPE-NPs (normal sera, NS) (bottom row). Red fluorescence portrays nanoparticle uptake in cells (DiI dye presence). Scale bar represents $200 \mu \mathrm{m}$. Magnification was at $10 \mathrm{X}$.

\footnotetext{
Supplemental Figure 4. HBPE-NPs pre-treated with NS show stable protein corona formation and uptake by breast cancer cells. Representative Cytation 5 microscopic images from the transwell (two-chamber) experiment. HUVECSs (upper chamber) were treated with DiI dye-encapsulated COOH-HBPE-NPs, HBPE-PEG-NPs, and NS-treated COOH-HBPE-NPs. The bottom chamber was seeded with MDA-MB-231 cells from
} 
which images of nanoparticle uptake were acquired. Red fluorescence portrays nanoparticle uptake by cells (DiI dye presence). Scale bar represents $200 \mu \mathrm{m}$. Magnification was at $10 \mathrm{X}$. 


\section{Figure S1}

A.

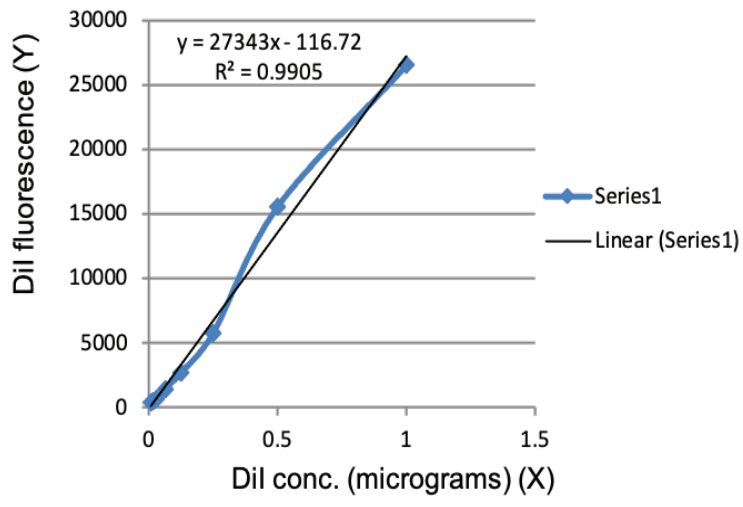

\begin{tabular}{|c|c|c|}
\hline Sample & $\begin{array}{c}\text { Fluorescence } \\
\text { avg + stdev (per } \\
50 \mu \text { Lolume) }\end{array}$ & $\begin{array}{c}\text { Dil } \\
\text { Concentration } \\
\text { per } 10 \mu l^{*}\end{array}$ \\
\hline $\begin{array}{c}\text { NP (Dil) } 10 \mu \mathrm{g} \\
\text { polymer }\end{array}$ & $786.33 \pm 44.836$ & $7863 \mu \mathrm{g}$ \\
\hline $\begin{array}{c}\text { NP (Dil)-PEG } \\
10 \mu g \text { polymer }\end{array}$ & $990.33 \pm 25.541$ & $9903 \mu \mathrm{g}$ \\
\hline
\end{tabular}

* Representative of typical dose (0.1 mg) per 50,000 cells

B.

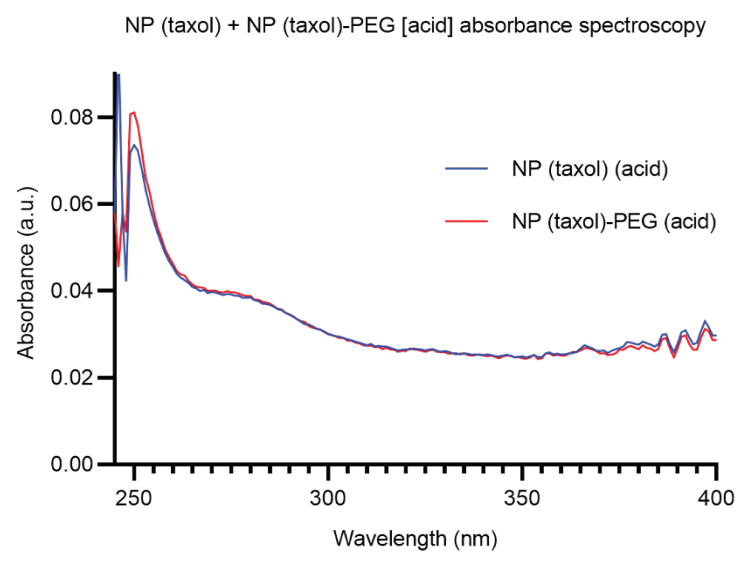

\begin{tabular}{c|l|c} 
Sample & $\begin{array}{l}\text { Absorbance (at } \\
250 \mathrm{~nm})(\text { per } 1 \\
\mathrm{ml} \text { volume) }\end{array}$ & $\begin{array}{c}\text { Taxol } \\
\text { Concentration } \\
\text { per } 1 \mathrm{ul}^{*}\end{array}$
\end{tabular}

$$
\begin{aligned}
& \text { NP (Dil) } 100 \\
& \mu g \text { polymer }
\end{aligned}
$$

NP (Dil)-PEG

$100 \mu \mathrm{g}$ polymer

* Representative of typical dose $(0.01 \mathrm{mg})$ per 50,000 cells

\begin{tabular}{|c|c|}
\hline $\begin{array}{c}\text { Sample } \\
\text { (Taxol Dilutions) }\end{array}$ & $\begin{array}{c}\text { Absorbance } \\
\text { average + stdev (at } \\
250 \mathrm{~nm} \text { ) (per } 1 \mathrm{ml} \\
\text { volume) }\end{array}$ \\
\hline Taxol $(5.4687 \mu \mathrm{g})$ & $0.0624 \pm 0.00311$ \\
\hline Taxol $(10.9375 \mu \mathrm{g})$ & $0.1159 \pm 0.00346$ \\
\hline Taxol $(21.875 \mu \mathrm{g})$ & $0.1921 \pm 0.00467$ \\
\hline Taxol $(43.75 \mu \mathrm{g})$ & $0.3655 \pm 0.00615$ \\
\hline Taxol $(87.5 \mu \mathrm{g})$ & $0.6404 \pm 0.0076$ \\
\hline
\end{tabular}


Figure S2

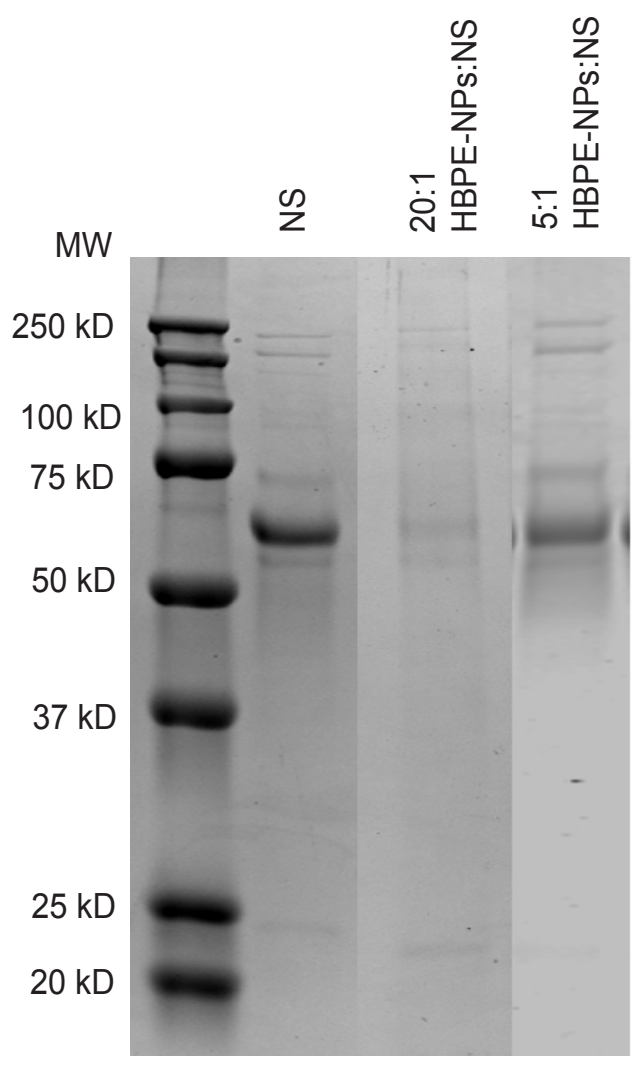


Figure S3

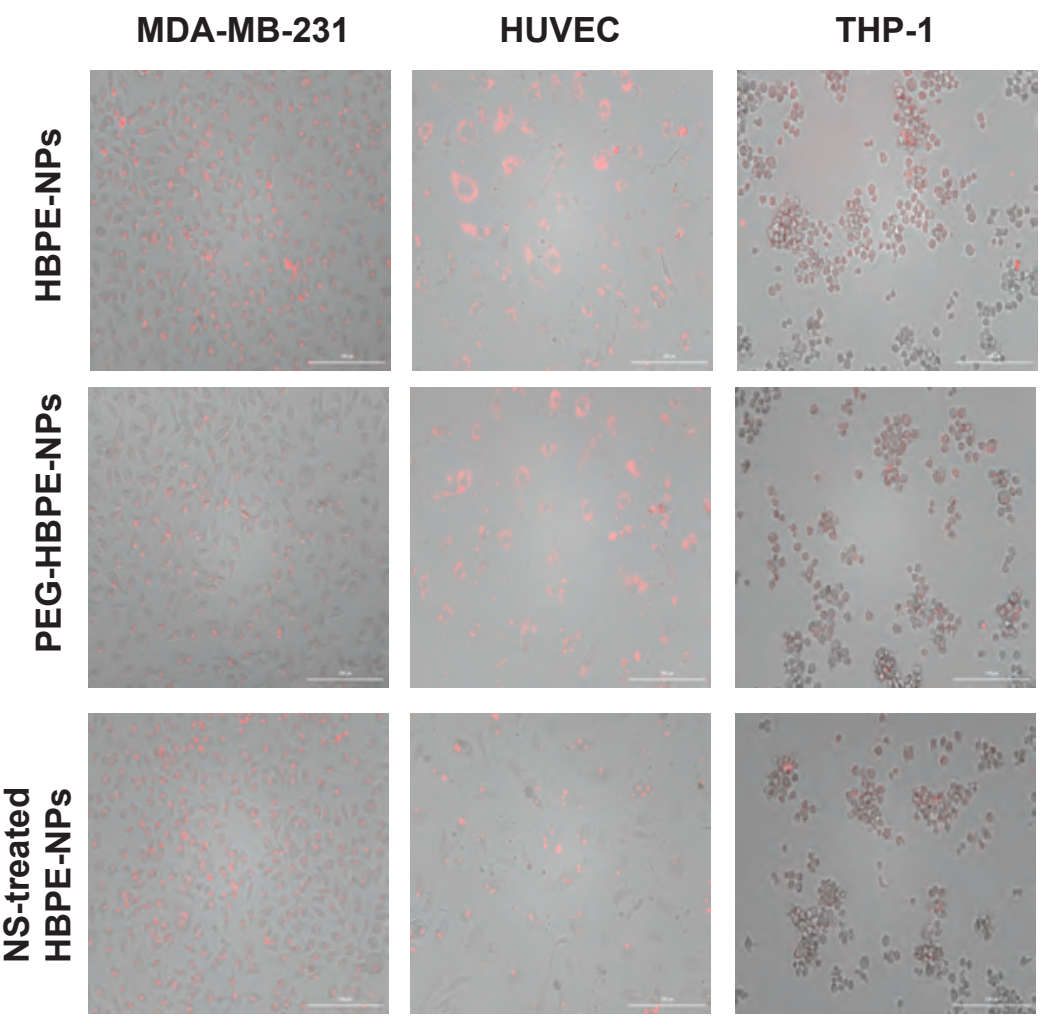


Figure S4

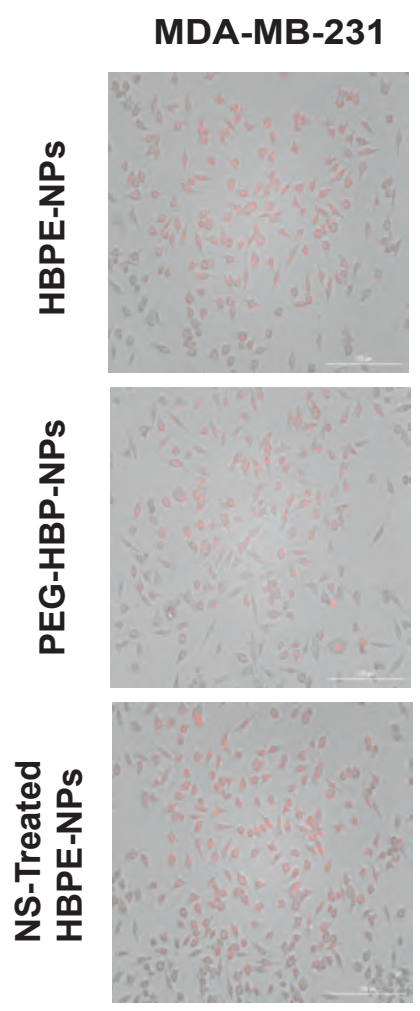

